\title{
Factor General de personalidad: historia de una aventura interdisciplinar
}

\author{
Antonio Caselles \\ Departament de Matemàtica Aplicada \\ Universitat de València \\ Valencia, Spain \\ Antonio.Caselles@uv.es
}

\author{
Joan C. Micó \\ Intitut Universitari de Matemàtica Multidisciplinar \\ Universitat Politècnica de València \\ Valencia, Spain \\ jmico@mat.upv.es
}

Salvador Amigó.

Departament de Personalitat, Avaluació i Tractaments Psicològics.

Universitat de València

Valencia, Spain

Salvador.Amigo@uv.es

\section{Presentación}

El Factor General de Personalidad (FGP) es un nuevo enfoque psicológico del estudio de la personalidad humana que se basa en la idea de que, como en el caso de la Inteligencia General, hay un súper-factor de personalidad que aglutina $\mathrm{y}$ representa a todos los demás aspectos de la personalidad. Por tanto, puede considerarse un sistema de subsistemas de personalidad, un sistema global e integrado de todos los diferentes componentes de la personalidad.

Así, el FGP puede ser estudiado desde la psicología y también desde la teoría de sistemas. En cuanto a la psicología, el abordaje teórico y empírico del FGP se ha limitado al estudio estructural, es decir, al estudio correlacional y estructural de los principales factores de personalidad y el FGP. Entre los modelos de personalidad que se han utilizado para realizar estos estudios destaca el modelo de los Cinco Grandes (Extraversión, Neuroticismo, Apertura a la Experiencia, Amabilidad y Responsabilidad) y el modelo de Eysenck
(Extraversión, Neuroticismo, Psicoticismo). Pero nada se sabe de la relación dinámica de esos factores a la hora de explicar el súper-factor de personalidad que representa el FGP.

El principal objetivo de nuestro grupo de investigación ha consistido en el estudio de la dinámica del FGP y de los factores que lo componen. Para ello, se ha partido de la Teoría del Rasgo Único de Personalidad [1] y de la teoría de sistemas, para avanzar en el estudio y la comprensión del FGP y diseñar un modelo matemático que explique su dinámica. Se han incluido en el modelo las drogas estimulantes como el estímulo que inicia la respuesta del organismo y, por tanto, del FGP.

En la actualidad, hemos podido constatar y describir la dinámica del súper-factor de personalidad del ser humano, a la vez que nos hemos adentrado en otras aéreas relacionadas, como es el estudio del cambio del FGP con Terapia de AutoRegulación (TAR), la personalidad de las sociedades, o el dilema cuerpo-mente. 
A continuación se explica con un poco más de detalle y de forma cronológica la evolución del trabajo del equipo a través de algo más de una década.

\section{Los comienzos: la Teoría del Rasgo Único de Personalidad y de la teoría de sistemas}

En el año 2005 Salvador Amigó publica el libro La Teoría del Rasgo Único de Personalidad, que se identifica con la Extraversión, en la que se sientan las bases científicas de la existencia de un suprasistema de personalidad, de un solo factor que aglutina a todos los demás sistemas de personalidad, además de presentar, por primera vez en este ámbito de estudio, la TAR para el cambio del rasgo único, a partir de la reproducción mental de los efectos de drogas. Por tanto, se admitía el supuesto de que las drogas pueden cambiar el rasgo único, es decir, la personalidad global en el corto plazo (mientras dura el efecto de la droga) y que se puede aprender a reproducir mentalmente el efecto de las drogas con TAR y, por tanto, cambiar temporalmente la personalidad global a voluntad.

Ese mismo año coincidimos los tres $\mathrm{y}$ mostramos el interés de colaborar en el proyecto de elaborar un modelo matemático dinámico para estudiar la dinámica del rasgo único a partir de la reacción de este rasgo ante el consumo de drogas estimulantes. Antonio Caselles y Joan C. Micó contaban con una amplia y extensa experiencia docente e investigadora en teoría de sistemas. Además, Caselles había desarrollado unos instrumentos matemáticos: SIGEM [2], para la programación automática de los modelos matemáticos complejos, REGINT [3], con el que buscar funciones de varias variables ajustadas a un conjunto de datos y PARDOSU [4] con el que ajustar sistemas de ecuaciones diferenciales a un conjunto de datos.

Los primeros meses sirvieron para aportar al grupo nuestros conocimientos y experiencia científica, que siempre es complejo cuando se trata de llevar a cabo una auténtica colaboración interdisciplinar desde campos muy diversos, y para organizar la agenda de trabajo. Ya desde el principio resultó ser una apuesta de alto nivel de dificultad, a la vez que productiva y apasionante.

\section{Primeras publicaciones: Dinámicas a corto y a largo plazo de la Extraversión}

En la primera publicación internacional se presentó el modelo matemático de Extraversión, en la que se mostró cómo el Rasgo Único o Extraversión reaccionaba a una dosis única de estimulante [5]. Si la Extraversión es una dimensión bipolar que se extiende desde el polo de la Extraversión extrema hasta el de la Introversión, vimos que podíamos clasificar a las personas a lo largo de ese polo en función de su reacción a una dosis de droga.

También elaboramos un modelo matemático dinámico para predecir la evolución de la Extraversión a lo largo de dos años con un consumo continuado de cocaína [6]. Por tanto, nuestro modelo era capaz de describir el efecto de una droga no solo de una dosis única sino de una secuencia en el tiempo de consumos repetidos.

\section{Sale en escena el Factor General de Personalidad}

Publicamos un estudio en el que se demuestra que la Extraversión, como Rasgo Único, es el súper-sistema que integra los principales factores de personalidad de los modelos de personalidad más influyentes, como el de los Cinco Grandes y el de Eysenck. [7]. En las diferentes revisiones del artículo tomamos conciencia de que el concepto de rasgo único estaba ya convirtiéndose en una poderosa línea de investigación internacional, pero que el nombre con el que se conocía este rasgo era el de Factor General de Personalidad. Si bien nosotros seguíamos manteniendo el mismo concepto de rasgo único y los mismos mecanismos de acción que lo explicaban, optamos por asumir el nombre con el que se le conocía internacionalmente, y empezamos a aceptar el término de Factor General de Personalidad en las siguientes publicaciones.

\section{Estudios experimentales con el Factor General de Personalidad (FGP): validación del modelo teórico y matemático}

Obtuvimos una escala breve de solo 5 ítems en formato-estado para evaluar el cambio del FGP [8]. De esta manera era posible medir la evolución del FGP como reacción a una dosis única de droga. 
Realizamos experimentos en los que los participantes tomaban una dosis de café y evaluamos tanto la reacción del FGP como de los Cinco Grandes $[9,10]$. Los resultados avalaron lo que ya el modelo teórico-matemático anunciaba, y es que es posible predecir la respuesta de los sujetos a los efectos a corto plazo de una droga. Estábamos en el camino de validar un modelo general de personalidad dinámico, lo que a nuestro entender era la primera demostración que se realizaba al respecto en la comunidad científica.

\section{Las bases biológicas de la dinámica del Factor General de Personalidad: estudios experimentales con metilfenidato}

En el proceso de validación de un modelo integral y dinámico del FGP nos propusimos estudiar el fundamento biológico. En un estudio experimental de caso único pudimos comprobar que el metilfenidato, una droga estimulante de uso en psiquiatría, producía un cambio en los niveles de glutamato en sangre en la línea de lo establecido en el modelo original, que designa al glutamato como el neurotransmisor por excelencia del FGP [11].

Por otra parte, en estudios de caso único pudimos validar nuestro modelo a partir de los cambios que el metilfenidato producía en dos genes reguladores: el DRD3 y el c-fos [12,13]. El DRD3 es un gen que regula los mecanismos inhibitorios celulares y lo contrario es cierto para el c-fos. En estos estudios pudimos constatar que, efectivamente, los mecanismos activadores e inhibitorios genéticos que observamos eran coherentes con nuestro modelo teórico-matemático.

\section{Aparece la Terapia de Auto-Regulación para reproducir mentalmente los efectos de las drogas}

En los estudios biológicos que se acaban de señalar [11-13] se empleó un procedimiento de sugestión diseñado por Salvador Amigó, llamado Terapia de Auto-Regulación (TAR), que enseña a "imitar" o reproducir los efectos de las drogas [14]. Se comprobó que la TAR modifica los parámetros biológicos (glutamato, DRD3 y c-fos) de la misma manera que lo hace la propia droga, además de reproducir los mismos efectos subjetivos y también la misma dinámica de la personalidad. Por tanto, contamos con un procedimiento que en futuros estudios puede sustituir a la toma de drogas.

En el Congreso Mathematical Modelling in Engineering \& Human Behaviour 2017 Conference, celebrado en julio de 2017, se presentó una comunicación en la que se confirmó que los mismos parámetros del modelo matemático dinámico que explican el efecto del metilfenidato en la personalidad eran aplicables cuando en lugar de la droga se utilizaba la TAR [15].

\section{Nos planteamos ahondar en la relación cuerpo-mente}

Los experimentos citados anteriormente que buscaban indagar en el sustrato biológico del FGP y su dinámica generaron una nueva línea de trabajo: el estudio de la relación cuerpo-mente. Este antiguo problema de la humanidad se trató dinámicamente, involucrando las bases biológicas de la personalidad, (en particular el gen c-fos y neurotransmisor glutamato) y el FGP, a través de ecuaciones en derivadas parciales, cuando el estímulo es el metilfenidato [16].

\section{Más allá del individuo: el Factor General de Personalidad en las sociedades}

La idea de que los mismos mecanismos subyacen en la dinámica de los individuos, grupos y amplios colectivos es consustancial a la teoría de los sistemas vivientes complejos [17]. Es por eso que hemos iniciado el camino para validar nuestro modelo teórico-matemático en las sociedades. En un primer momento propusimos un modelo teórico, basado en la teoría de sistemas, para validar la teoría de las supervivencia de las sociedades de Salvador Amigó [18]. Esta teoría sugiere una dinámica de la evolución de las sociedades como reacción a las crisis que atraviesan y en una publicación esbozamos una primera aproximación de un modelo teóricomatemático [19].

Más adelante, en una reunión científica en la Universidad Complutense de Madrid se presentó un modelo basado en una teoría más amplia de la personalidad global [20], inspirada en parte por la teoría de personalidad de Carl Jung y reelaborada por Amigó [21], con la pretensión de que fuera extrapolable a las sociedades. Se abría así la 
posibilidad de que nuestro modelo teóricomatemático de personalidad fuera también aplicable a las sociedades de cualquier lugar y época.

\section{A la búsqueda de un modelo unificador de la psicología y la física. Mecánica cuántica, personalidad y cerebro}

Hasta cierto momento de nuestra investigación los modelos desarrollados tenían al tiempo como única variable de referencia del cambio. Pero la realidad que nos planteamos es que el cerebro, como parte fundamental del sistema de estrés, sustrato fisiológico del FGP, está localizado en una zona espacial limitada. Por tanto, nos propusimos generalizar nuestro modelo matemático de respuesta de dosis única, incluyendo la variación espacial además de la temporal. Las investigaciones nos llevaron a un modelo matemático de respuesta espacio-temporal dado por una ecuación parabólica en derivadas parciales. Las condiciones de contorno del cerebro proporcionan una novedad sorprendente: un conjunto de resultados infinito numerable de funciones cuantizadas que se corresponden con un conjunto infinito numerable de valores propios también cuantizados, y que dependen de unos números enteros [22].

Este nuevo enfoque espacio-temporal proporciona unos resultados similares a los mecanocuánticos para el átomo de hidrógeno o para el cuerpo negro de Planck. En la actualidad intentamos profundizar en el mismo, por una parte, introduciendo experimentalmente el nivel tónico en el modelo como una función espacial, concepto aún no presente en [22], y por otra parte, buscando datos experimentales para su validación. Por tanto, un desarrollo espacio-temporal validado como el que proponemos equivaldría a un enfoque interdisciplinar que incluiría la física, el cerebro y la personalidad.

\section{El método como objetivo fundamental de nuestro trabajo}

En todo nuestro trabajo nos hemos basado en el método científico, la matemática compleja y la estadística inferencial. Hemos propuesto modelos teóricos y hemos realizado estudios empíricos con diseños experimentales de grupo y diseños de caso único. Pero desde el primer momento, y especialmente en el presente, nuestro interés por mejorar el método de trabajo ha sido una constante.

De hecho, la evolución de nuestra teoría ha sido una auto-exigencia conforme nos proponíamos enfrentarnos a nuevos retos. Por ejemplo, el modelo matemático de respuesta a una droga estimulante fue concebido en [5,9] como una ecuación diferencial de retraso discreto, más conocida en matemáticas como una ecuación en diferencias finitas. Sin embargo, ante el reto de enfocar el problema cuerpo-mente, el modelo pasó a ser una ecuación diferencial de retraso continuo en $[13,15,16]$, conocida en matemáticas como ecuación integro-diferencial. Además hemos de añadir a las ecuaciones obtenidas, la ecuación en derivadas parciales con la que se puede estudiar tanto el problema del cuerpo-mente [16] como la relación dinámica del FGP y los 5 Grandes [10]. Y para contemplar el conjunto del catálogo de ecuaciones, que son una generalización de las anteriores, tenemos el modelo espaciotemporal del cerebro [22], que podría arrojar luz matemática sobre les frecuencias fundamentales del electroencefalograma.

\section{Conclusión}

Hemos resumido en este artículo la evolución de nuestro trabajo interdisciplinar de más de una década. Habíamos empezado diciendo que el trabajo interdisciplinar serio es complicado, y así lo fue en un principio. Poco a poco nos fuimos adaptando a un sistema de trabajo organizado y fructífero que ha ido mejorando paulatinamente el trabajo conjunto auspiciado por una sinergia de grupo.

El resultado, hasta el momento, es que contamos con un modelo teórico-matemático, capaz de describir y explicar la dinámica de la personalidad completa y de sus más importantes componentes, a partir de los estímulos ambientales, especialmente las drogas, ya que la reacción a las mismas proporciona una información privilegiada, detectable y registrable, por la intensidad de la misma.

Además de conocer en mayor profundidad la dinámica de la personalidad global de los seres humanos, nos hemos adentrado en el estudio del dilema mente-cuerpo, y en el análisis del cambio voluntario y rápido de la personalidad utilizando la Terapia de Auto-Regulación, creada también por uno 
de nosotros.

Además, hemos llevado a cabo las primeras propuestas de validar nuestro modelo dinámico más allá de los individuos, para las sociedades, y hemos realizado un gran esfuerzo en mejorar el método de trabajo interdisciplinar, acercando la matemática compleja y la estadística inferencial, buscando puentes metodológicos de encuentro entre disciplinas tan aparentemente alejadas como la matemática y la psicología y, en definitiva, las llamadas ciencias físicas y la ciencias humanas.

Pero el camino se está todavía recorriendo, hay mucha tarea que realizar. Es por esto, que hemos querido con este número especial de la RIS, por una parte exponer nuestra trayectoria hasta el presente $\mathrm{y}$, por otra parte, ofrecer algunas de nuestras investigaciones más recientes.

En este número presentamos nueva confirmación sobre la estructura y dinámica del FGP, así como de la eficacia de la TAR para cambiar a corto y medio plazo la personalidad global, lo que abre un campo nuevo de importantes aplicaciones en psicología, psiquiatría y neurociencias. Por otra parte, también ofrecemos un modelo teórico desarrollado del Factor General de Personalidad de las sociedades, así como algunas sugerencias para su desarrollo matemático. También ofrecemos las nuevas aportaciones metodológicas en las que superamos el "viejo modelo de respuesta" y lo sustituimos por un modelo más eficaz y sensible, en la línea apuntada más arriba de nuestra firme intención de mejorar continuamente la metodología de trabajo.

En definitiva, este número especial sobre el Factor General de Personalidad y teoría de sistemas pretende ser no solo una presentación de lo realizado hasta ahora sino, sobre todo, una propuesta de futuro para seguir profundizando en el conocimiento de la naturaleza humana y en el desarrollo de un modelo integrador de los distintos campos del conocimiento, desde la física hasta la psicología, así como del individuo y la sociedad a la que pertenece.

\section{REFERENCIAS}

[1] Amigó, S. (2005). La teoría del rasgo único de personalidad. Hacia una teoría unificada del cerebro y la conducta [The Unique Personality Trait Theory. Towards a unified theory of brain and behavior]. Valencia, Spain: Universitat Politècnica de València.

[2] Caselles, A. (1994). Improvements in the Systems Based Program Generator SIGEM. Cybernetics \& Systems, 25, 81-103.

[3] Caselles, A. (2008). Modelización y simulación de sistemas complejos (Modeling and simulation of complex systems). Valencia, Spain: Universitat de València, (Available in http://www.uv.es/caselles (accessed 04.04.2017) as well as SIGEM and REGINT).

[4] Caselles, A., Micó, J. C. and Amigó, S. (2017). A genetic algorithm to calibrate systems: a case in Psychology. Proceedings of the Mathematical Modelling in Engineering \& Human Behaviour 2017 Conference. Valencia (Spain), September 17th to 19th, 2017.

[5] Amigó, S., Caselles, A., \& Micó, J. C. (2008). A dynamic extraversion model: the brain's response to a single dose of a stimulant drug. British Journal of Mathematical and Statistical Psychology, 61, 211231.

[6] Caselles, A., Micó, J. C., \& Amigó, S. (2010). Cocaine addiction and personality: A mathematical model. British Journal of Mathematical and Statistical Psychology, 63, 449-480.

[7] Amigó, S., Caselles, A., \& Micó, J. C. (2010). The General Factor of Personality Questionnaire (GFPQ): Only one factor to understand the personality? The Spanish Journal of Psychology, 13, 5-17.

[8] Amigó S., Micó J. C., \& Caselles A. (2009). Five adjectives to explain the whole personality: A brief scale of personality. Revista Internacional de Sistemas, 16, 41-43.

[9] Caselles A, Micó, C, and Amigó S. (2011). Dynamics of the General Factor of Personality in response to single dose of caffeine. The Spanish Journal of Psychology, 14, 675-692.

[10] Micó J. C., Amigó S., \& Caselles A. (2014). From the Big Five to the General Factor of Personality: a Dynamic Approach. Spanish Journal of Psychology, 17, 1-18. 
[11] Amigó S, Caselles A, Micó J. C. and García J. M. (2009). Dynamics of the unique trait of personality: blood's glutamate in response to methylphenidate and conditioning. Revista Internacional de Sistemas, 16, 35-40.

[12] Amigó S, Caselles A and Micó J. C. (2013). Self-regulation therapy to reproduce drug effects: A suggestion technique to change personality and DRD3 gene expression. The International Journal of Clinical and Experimental Hypnosis 61, 282-304.

[13] Micó J. C., Amigó S. and Caselles A. (2012). Changing the General Factor of Personality and the c-fos expression with methylphenidate and SelfRegulation Therapy. The Spanish Journal of Psychology, 15(2), 850-867.

[14] Amigó S. (1998). Self-regulation Therapy: Suggestion Without Hypnosis. In I. Kirsch, A. Capafons, E. Cardeña\& S. Amigó (Eds). Clinical Hypnosis and Self-Regulation Therapy: A cognitivebehavioral perspective. Washington: American Psychological Association.

[15] Amigó, S., Micó, J. C. \& Caselles, A. (2017). Methylphenidate and Self-Regulation Therapy: A systemic mathematical model. Proceedings of the Mathematical Modelling in Engineering \& Human Behaviour 2017 Conference. Valencia (Spain), September 17th to 19th, 2017.

[16] Micó, J. C., Caselles, A., Amigó, S., Cotolí, A. and Sanz, M. T. (2013). A Mathematical Approach to the Body-Mind Problem from a System Personality Theory (A Systems Approach to the Body-Mind Problem). Systems Research and Behavioral Science 30, 735-749.

[17] Miller, J. G. (1978). Living systems. New York: McGraw-Hill.

[18] Amigó, S. (2001). La teoría de la supervivencia de las sociedades. Hacia una teoría general de la evolución de la humanidad. Valencia, Spain: Promolibro.

[19] Amigó, S., Micó, J. C. y Caselles, A. y (2009).
Society survival theory and the contemporary age: proposal of a system approach. Revista Internacional de sistemas, 16, 44-48.

[20] Amigó, S. (2017). Una aproximación matemática a la evolución de las sociedades. Segundo Taller de Conferencias sobre Sociología y Matemáticas.

[21] Amigó, S. (2014). El enigma de la conciencia. Entre la razón, la intuición y el misterio. Madrid: Liber Factory.

[22] Micó, J. C. and Sanz, M. T. (2013). A spatiotemporal approach to brain dynamics. Revista Internacional de Sistemas, 18, 29-38 (Available in http://www.uv.es/caselles/). 